\title{
ㄷำ1
}

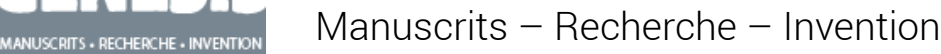

$37 \mid 2013$

Verbal - Non verbal

\section{Penser avec}

\section{Marie-Haude Caraës et Nicole Marchand-Zanartu}

\section{(2) OpenEdition}

\section{Journals}

Édition électronique

URL : http://journals.openedition.org/genesis/1250

DOI : 10.4000/genesis. 1250

ISSN : 2268-1590

\section{Éditeur :}

Presses universitaires de Paris Sorbonne (PUPS), Société internationale de génétique artistique littéraire et scientifique (SIGALES)

\section{Édition imprimée}

Date de publication : 15 décembre 2013

Pagination : 157-162

ISBN : 9782840509196

ISSN : $1167-5101$

\section{Référence électronique}

Marie-Haude Caraës et Nicole Marchand-Zanartu, « Penser avec », Genesis [En ligne], 37 | 2013, mis en ligne le 21 mars 2016, consulté le 21 janvier 2021. URL : http://journals.openedition.org/genesis/1250 ; DOI : https://doi.org/10.4000/genesis. 1250

Ce document a été généré automatiquement le 21 janvier 2021.

Tous droits réservés 


\title{
Penser avec
}

\author{
Marie-Haude Caraës et Nicole Marchand-Zanartu
}

Il est facile d'apercevoir un champignon. Mais

lequel?

Il y faut toujours une sorte de révélation. Une

vision doit

traverser les paroles et les imageries et nous

transporter

dans un monde irrésistible où s'affirme enfin

dans son

incroyable vérité le champignon vivant. Enfin

n'oubliez

pas qu'ils ne poussent jamais sans un

saisissement.

André DHÔTEL, La Rhétorique fabuleuse

1 «Comment n'y avons-nous pas pensé plus tôt », fut la conclusion de notre éditeur quand nous lui proposâmes notre livre ${ }^{1}$. Un corpus constitué de soixante-quatre images puisées dans tous les champs du savoir, du XIII ${ }^{\mathrm{e}}$ au XXI ${ }^{\mathrm{e}}$ siècle. Révéler cet objet d'étude, ce sous-sol de la pensée quand elle affleure, a été notre impératif. À l'étude critique qui à ce stade de la découverte ne faisait pas sens, nous avons préféré situer chaque image dans son contexte de création et raconter la constitution de cet objet de recherche, ce que l'on pourrait appeler la « cuisine de la pensée », laissée trop souvent dans l'ombre des recherches. Permettre à chacun de plonger son regard et se perdre peut-être dans le labyrinthe de ces images.

2 La réception de cet ouvrage nous a encouragées à continuer notre quête : actuellement plusieurs dizaines d'images nous conduisent à nous interroger sur les formes particulières de l'imagination graphique quand celle-ci prend naissance en même temps que la pensée. Ce sera l'objet d'un second volume.

3 Les représentations graphiques ont toujours accompagné les grands courants de la pensée : arbres de la connaissance, planches anatomiques, cartes et plans de toutes sortes, ont figuré le monde pendant des siècles. Mais en marge de ces figures, à côté des 
métaphores et symboles d'une époque et de leur clé d'interprétation, à côté du cercle, du filet, de l'arbre, du labyrinthe, du plan, de l'océan, de la carte, de maladroites représentations, des gribouillages vont par un chemin de traverse dire le monde autrement. Ce sont de simples dessins et à première vue nous serions tous capables de les exécuter. Tracées à l'abri du regard, ces figures aux formes variées cachent en réalité, dans leurs plis, le travail de la pensée en son moment le plus matinal et le plus secret. Cette saisie en un regard spatial, comme prise de l'intérieur, dans le creuset même de sa formation, ou plutôt dans son aspiration à devenir, prend naissance dans des sphères qui échappent au langage. Savoir, imagination, vision, intuition, pressentiment, rêverie, illumination, entrent dans la composition de ces images où faisceaux, indices sont assemblés dans une forme qui n'a peut-être pas toujours grande valeur en soi mais le pouvoir de faire surgir l'impensable de la création. À cet ensemble baroque de formes remuantes, nous avons donné le nom d'« images de pensée ».

Ce n'est pas le célèbre divan qui figure sur la carte postale de la Sigmund-Freud-Haus rapportée de Vienne par un ami, mais un simple schéma au titre troublant: Sexualschema, 1895 (voir ici même). Est-ce une île, le cours d'une rivière, un plan secret, qui se cache dans ce contour tremblé parcouru de flèches rouges et noires, de boucles, de pointillés? Une intensité traverse ces quelques traits, un mouvement, une circulation. Le trouble s'installe. Et ces mots en allemand qui ne nous aident guère. Tout se voile en un rébus, une butée. Nous découvrons qu'il s'agit du schéma de la sexualité dans la mélancolie qui figure dans le Manuscrit $G$ que Freud envoie à son ami berlinois le psychiatre Wilhelm Fliess, longtemps son confident. Freud a trente-neuf ans, c'est un spécialiste des maladies dites «nerveuses». La psychanalyse n'existe pas encore. Ce n'est pas le premier schéma que dessine Freud, mais celui-ci est d'une facture différente, un condensé de deux univers pensés d'un seul coup d'œil dans l'espace. Cet effort de construction graphique, cette capture en une image unique permet à Freud de tenir l'écheveau multiforme de sa pensée et de faire émerger «le mouvement circulaire de la tension sexuelle» qui le conduira à distinguer «la mélancolie de la neurasthénie et la névrose d'angoisse ». Combien de pages faudra-t-il pour dévoiler ce long cheminement et dénouer la condensation de cette opération graphique, dans laquelle, selon l'interprétation psychanalytique, se cachent «le linéaire du signifiant et la structure circulaire de la pulsion ${ }^{2}$ ».

Est-ce l'énigme de la représentation, la tension de tous les éléments reliés entre eux, le recours de l'auteur au dessin ou tout cela réuni qui fascina notre regard et donna à notre quête - des dizaines de micro-mondes rassemblés dans tous les champs du savoir - son envol ? Ou le pressentiment que se cachait là une autre histoire de la pensée en marge de la grande histoire, un continent enchanté et aride à la fois, qu'une vie ne suffirait pas à épuiser.

\section{À l'ombre, toujours à l'ombre}

Elles se plaisent à l'ombre, cachées dans les replis du savoir. Trouver les images de pensée ressemble à un jeu où colin-maillard tenterait d'attraper cache-tampon. Une fois apprivoisées, il est difficile de les faire séjourner dans la même maison: leur caractère indépendant, leur physique atypique, leur époque, leur langage, semblent résister à toute forme de classement. Sorties des limbes, elles transcendent les codes de leur discipline et de leur époque et vont représenter le monde non pas tel qu'il existe 
mais dans une réalité non encore advenue. Leurs formes, mélange d'analytique et de subjectif, se déploient de façon inattendue sur une petite surface, celle d'une feuille, d'un carnet, d'un cahier, où se mêlent à un bric-à-brac personnel, des usages de géométries bricolées, où jouent des figures élémentaires : cercle, ligne, points, triangle, carré, boucle, spirale, etc. Une géométrie qui ne géométrise pas, mais transpose, métaphorise et cherche à rendre un espace intérieur. Inscrites dans une temporalité toujours décalée, les images de pensée dans leur condensation et leur retenue nous libèrent des images fermées que nous consommons chaque jour, nous sortent du flux iconographique dans lequel nous sommes plongés. Elles creusent en profondeur pour construire à partir d'indices la combinatoire secrète des mouvements de la pensée quand celle-ci se met en branle ou tend vers une résolution. Mélange d'assurance et de doute, de découverte et de révélation. Un arrêt sur image qui contient tout le film présent ou à venir. Comment nous y prendre pour pénétrer dans ce sous-bois et observer ces espèces inconnues? Ne devons-nous pas accepter de perdre nos repères pour nous replacer au cœur de chaque image, inventer une syntaxe, en soulevant chaque élément de leur composition avec la précaution et la patience d'un joueur de jonchet? Entrer en deçà des conventions du savoir dans un état que la phénoménologie nomme " ambiguïté perceptive », mélange de curiosité, d'ingénuité et de gai savoir.

Et si une part de leur fascination tenait dans ce tour de passe-passe qui donne à voir la complexité du monde avec presque rien, et que ce presque rien tienne debout tout seul, comme dans une œuvre d'art? «Tenir debout tout seul, ce n'est pas avoir un haut et un bas, ce n'est pas être droit [...], c'est seulement l'acte par lequel le composé de sensations créé se conserve en lui-même ${ }^{3}$.»

Une saturation de sens rendue en une ellipse. Sans être savants, ne faisons-nous pas tous cette expérience anthropologique à des degrés divers pour bâtir le monde dont nous faisons notre demeure? N'avons-nous jamais jeté quelques traits sur le papier pour dire un chemin, un espace, une maison, un rêve, le " je vais vous faire un dessin ", premier langage de l'enfance? Comme dans les mystères, nous sommes si près et si loin, si intimement requis par ces fragiles tracés accomplis dans le secret et la solitude, et qu'il s'agit de faire parler en compagnie de ces hommes qui nous convient à penser avec eux: le tracé rouge et bleu de la cinémathèque du jeune Langlois (fig. 2), le parachute de monsieur de Montgolfier bâti avec quelques effets de couturière, le va-etvient obligé de la pensée entre ordre et désordre de Philippe de Jonckheere, la simple et révolutionnaire soustraction de Dimitri Mendeleïev, l'arbre de la philosophie de Witold Gombrowicz, le bonhomme rouge d'André-Marie Ampère ou les premières esquisses de la Carte de la stratégie de la pauvreté dessinées sur une nappe en papier par Philippe Rekacewicz (fig. 3). Et le Strumpband (jarretière) de Gottfried Leibniz - nom sans doute emprunté au prestigieux ordre de la jarretière et à son ruban - dont la démonstration $\mathrm{du}$ nouage nous fait entrer, topologiquement, non pas dans le vol d'une chauve-souris dont il a l'allure, mais dans le concept de connaissance confuse et de la connaissance distincte. L'image de pensée est cependant injuste envers les femmes - et le constat attriste -, leurs travaux sont peu ou tardivement archivés. De même pour les dessins plus modestes, plus anonymes dont les traces ne subsistent que grâce à la passion de ceux qui les ont reconnues, protégées, déposées. 


\section{Dessiner à vue}

9 Jean Lauxerois attire notre attention sur «la longue tradition qui nous aurait accoutumés à séparer la pensée, l'image et l'écriture en valorisant la première aux dépens des deux autres ${ }^{4} »$. Il faut attendre la venue des romantiques anglais au $\mathrm{XVIII}^{\mathrm{e}}$ siècle, John Keats, Stephen Woodworth, Samuel Coleridge pour que l'imagination trouve sa majesté avant que Charles Baudelaire ne la transforme en "reine des facultés ». Au Xxe siècle, philosophes, écrivains, scientifiques, théoriciens de l'art, psychanalystes, vont se presser au chevet de l'imagination et ausculter son cœur énigmatique. Gaston Bachelard formulera la théorie de l'action imaginante : «On veut toujours que l'imagination soit la faculté de former des images. Or elle est plutôt la faculté de déformer les images fournies par la perception, elle est surtout la faculté de nous libérer des images premières, de changer les images. S'il n'y a pas changement d'images, union inattendue des images, il n'y a pas imagination, il n'y a pas d'action imaginante ${ }^{5}$. " Pour Henri Focillon, cette vie intérieure est un jeu de règles cachées qui se développe «sur des plans multiples, reliés par des passerelles, par des couloirs, par des degrés. [...] La vie des formes, sans cesse renouvelée, ne s'élabore pas selon des données fixes mais engendre diverses géométries, à l'intérieur de la géométrie même, comme elle se crée les matières dont elle a besoin ${ }^{6}$." François Dagognet verra dans l'imagination graphique un "instrument heuristique privilégié, une véritable néoécriture », capable, à elle seule, de transformer l'univers et de l'inventer. S'appuyant sur les dessins d'Étienne-Jules Marey, de La Méthode graphique dans les sciences expérimentales (1878), Dagognet proposera les termes d'imagination "inscriptive et abréviative » qui éclairent « la loi paradoxale de la représentation : plus est dépouillé, léger, nerveux, le graphisme inscripteur - plus il risque de traduire l'inaccessible, les aspects les plus enfouis de la réalité ${ }^{7}$ ». Gilles Deleuze, reprenant la figure botanique du rhizome « d'entrecroisement de forces ou de lignes où chaque chose a sa géographie, sa cartographie, son diagramme ", abolira en quelque sorte la vision structuraliste; ce qui compte, dira-t-il, « ce ne sont pas les débuts ni les fins, mais le milieu. Les choses et les pensées poussent ou grandissent par le milieu, et c'est là qu'il faut s'installer ${ }^{8}$ ».

Installons-nous dans ce milieu, là où le guetteur peut surprendre les images de pensée qui, comme les fleurs de la petite Ida d'Andersen, ne s'animent que la nuit, à l'abri des regards. 
Fig. 1

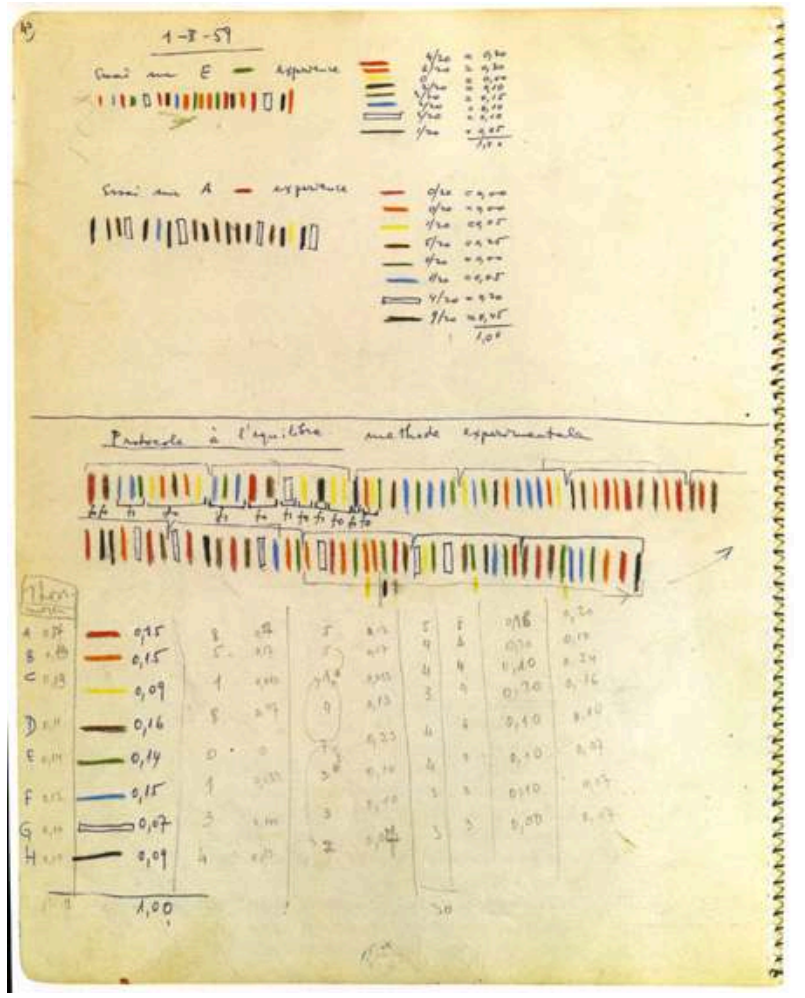

Fig. 1 : lannis Xenakis, compositeur, 1959 (BnF - Images de pensée, p. 101)

Fig. 2

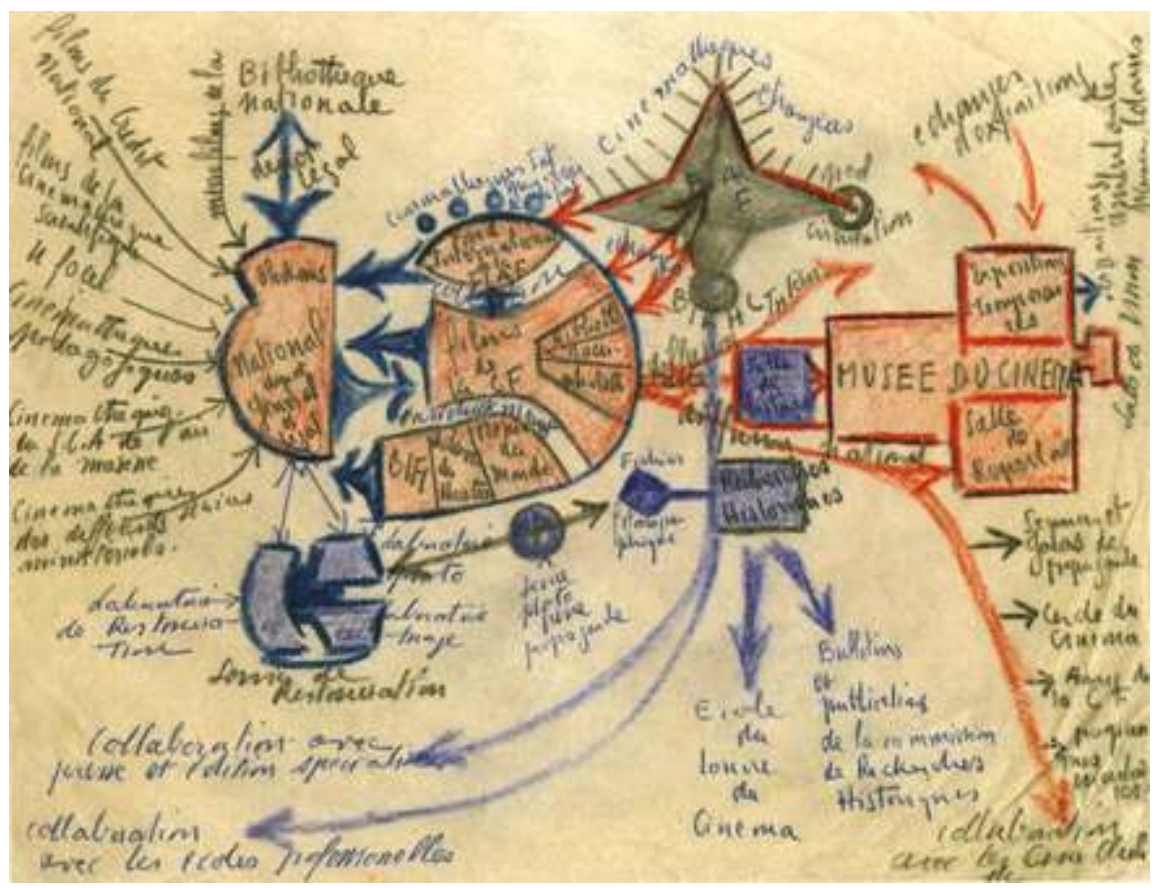

Fig. 2 : Henri Langlois, conservateur, schéma pour la création de la Cinémathèque française, 1934 (La Cinémathèque française, Paris - Images de pensée, p. 89) 
Fig. 3

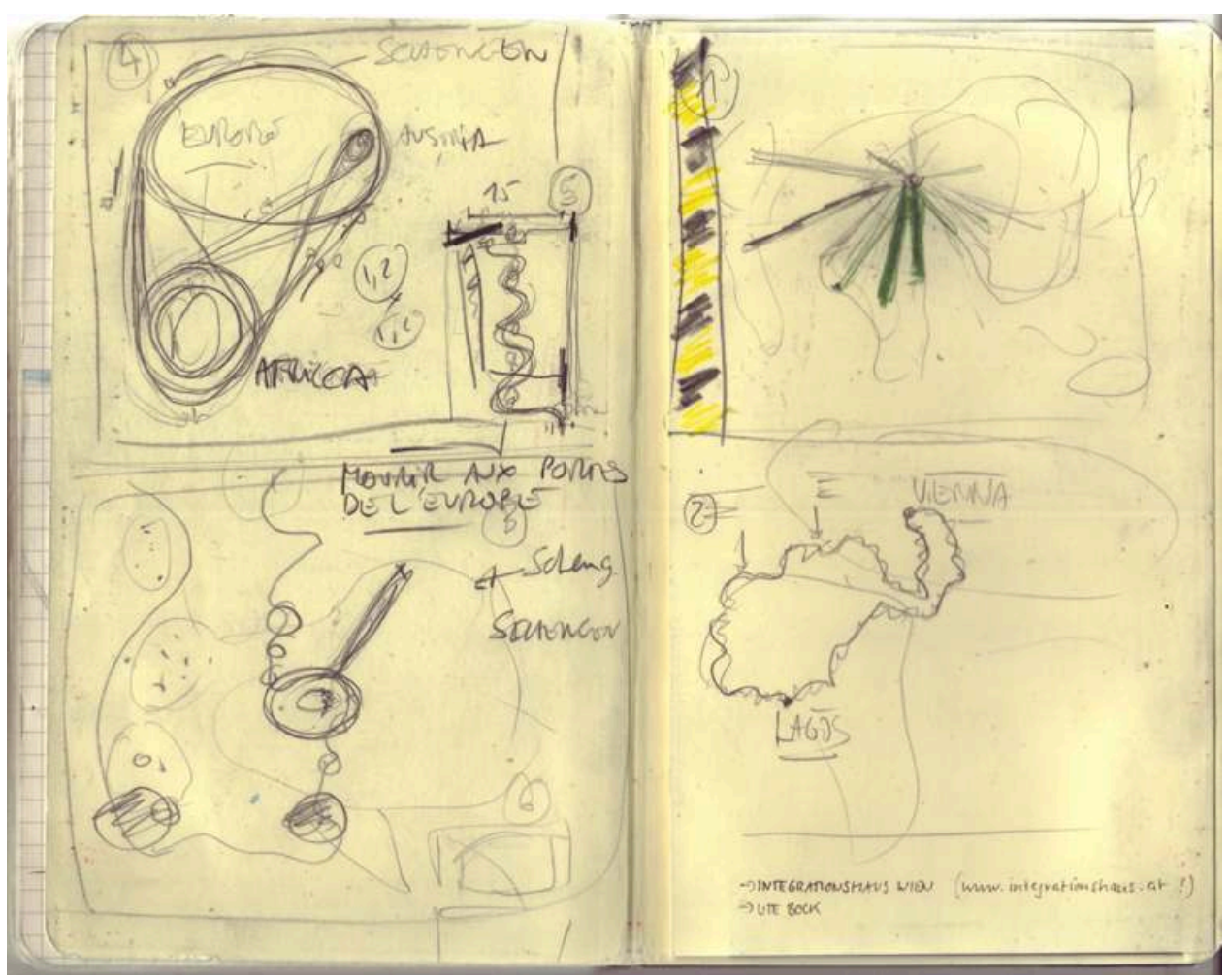

Fig. 3 : Philippe Rekacewicz, géographe, « Mourir aux portes de l'Europe » (2009) Premières esquisses de la Carte de la stratégie de la pauvreté. La grande roue (coll. particulière) - Images de pensée, p. 31)

\section{NOTES}

1. Images de pensée, Paris, RMN, 2011.

2. Dominique Inarra, "Pulsion et raison graphique ", Figures de la psychanalyse, $\mathrm{n}^{\circ} 5,2 / 2001$, p. 115.

3. Gilles Deleuze, Félix Guattari, Qu'est-ce que la philosophie ?, Paris, Minuit, 1996, p. 155.

4. Jean Lauxerois, «Éloge de l'imagination graphique », dans Images de pensée, op. cit., 2011, p. 115.

5. Gaston Bachelard, L'Air et les songes : essai sur l'imagination du mouvement, Introduction, Paris, José Corti, 1963, p. 5-6.

6. Henri Focillon, Vie des formes, Paris, PUF, 1964, p. 49.

7. Ibid., p. 77.

8. Gilles Deleuze, Pourparlers, Paris, Minuit, 1990, p. 219. 